\title{
The growth and expansion of meningeal lymphatic networks are affected in craniosynostosis
}

1 Phillip Ang ${ }^{1,2}$, Matt Matrongolo ${ }^{2,3}$, Max A Tischfield ${ }^{1,2^{*}}$

$2{ }^{1}$ Department of Cell Biology and Neuroscience, Rutgers University, Piscataway, NJ, USA

$3{ }^{2}$ Child Health Institute of New Jersey, Robert Wood Johnson Medical School, New Brunswick, NJ, 4 USA

$5 \quad{ }^{3}$ Human Genetics Institute of New Jersey, Piscataway, NJ, USA

6 * Correspondence:

7 max.tischfield@ rutgers.edu

8 Keywords: meningeal, lymphatic, craniosynostosis, Twist1, dural, venous sinuses

9 Word Count:

10 Figure Count: Four main figures, four supplemental figures

\section{Abstract}

12 Congenital skull malformations are associated with vascular anomalies that can impair fluid balance

13 in the central nervous system. We previously reported that humans with craniosynostosis and

14 mutations in TWISTI have dural venous sinus malformations. It is still unknown whether meningeal

15 lymphatic networks, which are patterned alongside the venous sinuses, are also affected. Using a

16 novel skull flat mounting technique, we show that the growth and expansion of meningeal lymphatics

17 are perturbed in Twistl craniosynostosis models. Changes to the local meningeal environment,

18 including hypoplastic dura and venous malformations, affect the ability of lymphatic networks to

19 sprout and remodel. Dorsal networks along the transverse sinus are hypoplastic with reduced

20 branching. By contrast, basal networks closer to the skull base are more variably affected, showing

21 exuberant growth in some animals suggesting they are compensating for vessel loss in dorsal

22 networks. Injecting molecular tracers into cerebrospinal fluid reveals significantly less drainage to the

23 deep cervical lymph nodes, indicative of impaired lymphatic function. Collectively, our results show

24 that meningeal lymphatic development is hindered in craniosynostosis, suggesting central nervous

25 system waste clearance may be impeded. 


\section{Introduction}

28 The development and functions of meningeal lymphatic vessels (mLVs) have become subjects of

29 intense interest because they are implicated in modulating the immunological state of the central

30 nervous system (CNS) (Alves de Lima et al., 2020). These vessels reside in dura mater, the

31 outermost meningeal layer encasing the CNS, along both the dural venous sinuses and meningeal

32 arteries (Antila et al., 2017). Though the putative functions of para-arterial mLVs have yet to be

33 discerned, perisinusoidal lymphatics along the venous sinuses have been found to uptake

34 cerebrospinal fluid (CSF) (Louveau et al., 2018a, Ahn et al., 2019). During steady state, mLVs act in

35 conjunction with the glymphatic system to transport macromolecules (Louveau et al. 2015), toxic

36 brain metabolites (Xie et al. 2013, Lundgaard et al. 2016), amyloid-beta plaques (Da Mesquita et al.,

37 2018), and dendritic cells (Louveau et al. 2018a) from the cranium to the deep cervical lymph nodes

38 (dcLNs). As such, mLVs are essential for CNS homeostasis and normal brain functions, and their

39 dysfunction is associated with neurodegeneration, cognitive impairment, and changes to

40 neuroinflammatory processes (Da Mesquita et al., 2018; Louveau et al., 2018a; Alves de Lima et al.,

41 2020).

42 Given their unique anatomical location, mLVs evaded our attention for centuries until their

43 "rediscovery" in 2015 (Aspelund et al., 2015; Louveau et al., 2015). mLV networks have now been

44 reported in humans, non-human primates, and rodents (Absinta et al., 2017). In mice, mLVs have a

45 defined postnatal developmental pattern that involves endothelial cell migration, tube formation, and

46 sprouting. mLVs start their growth by penetrating the dura through foramina at the base of the skull

47 and begin forming preliminary networks along both the meningeal arteries and dural venous sinuses

48 (Antila et al., 2017). As these networks mature, they continue to extend in a basal to dorsal direction.

49 Para-arterial mLVs begin at the pterygopalatine artery and complete their growth near the top of the

50 middle meningeal artery (MMA) by P28. Meanwhile, perisinus lymphatic networks begin developing

51 along both the sigmoid (SgS) and petrosquamosal sinuses (PSS), before ascending the transverse

52 sinus (TVS). Their development is completed by approximately P30, by which time coverage along

53 the superior sagittal sinus (SSS) is complete (Antila et al., 2017; Izen et al., 2018).

54 These vessels share many commonalities with peripheral lymphatics, including the expression of

55 Prox1, Lyve-1, and Podoplanin. In addition, mLVs also rely on Vegf-c/Vegfr3 signaling for their

56 growth and maintenance (Antila et al., 2017). Vascular smooth muscle is postulated to be a local 
source of Vegf-c and the growth of mLVs along blood vessels is concurrent with venous smooth muscle development (Antila et al., 2017). By contrast, differential gene expression in meningeal versus peripheral lymphatic vessels has revealed that mLVs are unique according to gene pathways related to extracellular matrix (ECM) interactions, focal adhesion, angiogenesis, and responses to endogenous and exogenous stimuli (Da Mesquita et al., 2018). Thus, processes that govern the growth and maturation of mLVs are likely specific to their unique meningeal environment. Although the detailed mechanisms of mLV development remain largely unknown, these likely include tissuespecific interactions with blood vessels and dura. Utilizing animal models that possess structural changes to dura mater and/or the resident venous vasculature should help facilitate the discovery of these processes and their specific regulation of mLVs.

We recently reported that humans with craniosynostosis caused by mutations in the transcription factor TWIST1 (Saethre-Chotzen syndrome) have dural venous sinus malformations, including loss and/or hypoplasia of the TVS, SgS, and jugular veins (Tischfield et al., 2017). Modeling the disorder in mice by inactivating Twist 1 in the cranial sutures and dura via Sm22a-Cre (Twist1 ${ }^{\text {Flx/Flx }}$ :Sm22aCre) unexpectedly revealed that Twistl regulates the growth and remodeling of the dural venous sinuses in a non-cell autonomous manner. It does so by promoting the development of osteoprogenitor cells and dura from cranial suture-derived mesenchymal progenitor cells, and these tissues produce growth factors (i.e. Bmp2/Bmp4) that act on the underlying venous endothelium (Tischfield et al., 2017). In addition to skull and vascular malformations, we also reported that dura was hypoplastic in affected embryos, but our analyses were limited to early embryonic stages preceding bone mineralization and skull development.

In the present study, we leverage Twist1 craniosynostosis models that possess venous malformations and structural changes to dura to gain insight into processes that regulate the growth and remodeling of mLV networks. Using a novel skull flat mounting technique, we show that the growth and expansion of mLV networks is perturbed in craniosynostosis. Vessels that grow along the TVS show reduced branching and loss of network complexity. By contrast, lymphatic networks that grow along the SgS and PSS near the skull base are less affected and, in some animals, are hyperplastic with long vessels and numerous sprouts. Additionally, infusing tracer compounds into CSF reveals less drainage to the dcLNs, suggesting meningeal lymphatic networks are functionally compromised in craniosynostosis. Thus, mouse models for craniosynostosis can provide unique insights into 
individuals with craniosynostosis may be at risk for impairments to meningeal lymphatic vessels and CNS waste clearance.

\section{Results and Discussion}

91 We generated craniosynostosis models by inactivating both copies of Twist 1 in cranial mesenchyme, as previously reported (Tischfield et al., 2017). Sm22a-Cre is expressed by e10.5 in neural crest and cranial mesoderm, giving rise to dura and arachnoid tissue (El-Bizri et al., 2008, Tischfield et al., 2017). Lineage labeling also shows strong Cre activity in sutural mesenchyme, especially the coronal and sagittal sutures (Fig. 1A). These regions of non-ossified tissue separate the paired frontal and parietal bones, give rise to osteoblasts, and are commonly affected in Saethre-Chotzen syndrome (Twigg and Wilkie, 2015; Ottelander et al., 2021). To analyze skull malformations in higher detail, the skulls were imaged using three-dimensional x-ray microscopy. This revealed varying degrees of suture fusion coupled with calvarial bone loss in most animals (Fig. 1B), and skull length from the nasal to occipital bones was reduced by $\sim 25 \%$ (Fig. S1). In a small subset (13\%, 4/30), the skull was intact and the animals had bilateral fusion of the coronal sutures and small dome shaped skulls. In $\sim 60 \%$ of animals (17/30), the coronal sutures showed bilateral fusion and bone loss was present along the dorsal midline where the sagittal suture is normally present, affecting the frontal, parietal, and interparietal bones (Fig. 1B). Areas that lacked proper mineralized bone instead contained a hardened, thin translucent material that lacked blood vessels but covered the underlying soft tissue. Also, focal bone loss was often observed in the region where the coronal suture is normally located (Fig. 2B). In the remaining animals, bone loss was more severe and stretched further away from the midline, and holes in the skull were sometimes present (Fig. S1). Thus, loss of Twist1 in sutural mesenchyme via Sm22a-Cre models a severe form of craniosynostosis marked by premature fusion and/or partial agenesis of the cranial sutures, which can lead to regionalized bone loss.

111 Periosteal dura is a thin portion of the dural membrane that directly underlies developing calvarial

112 bones and sutural mesenchyme, and it secretes growth factors that are critical for skull development

113 and suture maintenance (Ito et al., 2003; Lee et al., 2006). The dural venous sinuses and meningeal

114 lymphatic vessels also reside in periosteal dura. We therefore examined the development of dura at or 115 adjacent to the dorsal midline in e16.5 embryos, by which time the meninges have differentiated and 116 bone mineralization is commencing. 
117 In typical animals, the differentiation of the meninges starts at the skull base around embryonic day

11812.5 (e12.5) and proceeds until e14.5, by which time the three layers (dura, arachnoid, and pia) are

119 observed from the skull base to the apex. Similarly, osteoblasts are formed from intramembranous

120 ossification of condensed mesenchyme adjacent to dura. This process starts at eye level near the base

121 of the head at e12.5 and proceeds to the apex by e14.5-e15 (Deckelbaum et al., 2012; DeSisto et al.,

122 2020). In affected embryos, meningeal tissue closer to the skull base was relatively normal and three

123 layers were recognized. In severely affected embryos, however, condensed mesenchyme and dura

124 was largely absent along the dorsolateral margins of the head and dorsal midline, and the apical

125 expansion of bone growth was halted. The dural extracellular matrix was missing as fibronectin

126 staining was largely absent (Figs. 1C, D). In more common, mildly affected embryos, the dura and

127 meninges were intact but became noticeably hypoplastic at or near the dorsal midline. Unlike

128 controls in which condensed osteogenic mesenchyme was clearly separated from the overlying

129 dermis, these embryos showed less periosteal dura and osteogenic mesenchyme without clear

130 separation from the overlying dermis (Figures 1E, F). The superior sagittal sinus (SSS) was

131 hypoplastic but still enveloped by dura, and all affected animals had segmental or complete unilateral

132 loss of the TVS (Fig. S2), as reported previously in mice and humans (Tischfield et al., 2017).

133 Arachnoid tissue was also present, as detected by the presence of e-cadherin and connexin-43, but

134 fibronectin expression was reduced in regions where dura was hypoplastic, and n-cadherin positive

135 osteoblasts had not yet developed (Figs. 1G-I). These results show that calvarial bone loss parallels

136 the absence of dura, and loss of periosteal dura is more significant dorsolaterally and/or at the dorsal

137 midline of the head where the dural venous sinuses and mLV networks reside.

\section{Meningeal lymphatic networks are affected in Twist1 craniosynostosis models}

139 Typically, mLVs are examined by carefully scraping dura off the skull, followed by

140 immunohistochemistry, flat mounting, and imaging (Louveau et al., 2015). This was unfeasible in

141 Twist $1^{F l x / F l x}:$ Sm $22 a-C r e\left(\right.$ Twist $1^{C S}$ ) animals since the hypoplastic dura did not stay intact during

142 scraping. We instead removed the dorsal half of the skull, performed chromogenic

143 immunohistochemistry to stain vessels with Lyve-1, and imaged the lymphatic networks under a

144 stereomicroscope. In control animals, mLVs were patterned along the SSS and TVS as reported

145 (Antila et al., 2017). The networks were more complex with numerous branches at the sinus

146 confluence and at particular locations along the TVS (Fig. 2A, A'). These regions are proposed to be

147 "hotspots" specialized for the uptake of CSF (Louveau et al., 2018a). By contrast, meningeal 
148 lymphatic networks were poorly developed in Twist $1^{C S}$ animals (Figs. 2B, B', n=3). Networks along

149 the TVS appeared less complex and/or atrophic, and growth towards the sinus confluence was

150 impeded. Vessels along the SSS were also hypoplastic and/or missing (Fig. 2B'). Lineage labeling

151 showed that Sm22a-Cre is not expressed in mLVs (Fig. 2C). Furthermore, we previously reported

152 that Twist 1 is not expressed in meningeal endothelial cells, and Twist $1^{\text {FLX/FLX }}$ :Tie2-Cre embryos have

153 normal vascular development with no signs of edema (Tischfield et al., 2017). The development of

154 lymphatic networks in other tissues, such as ear skin, was normal (Fig. 2D). Thus, the observed

155 phenotypes are non-cell autonomous and are due to changes in the local environment that hinder the

156 proper growth and expansion of lymphatic networks.

157 The growth of meningeal lymphatic networks is perturbed in Twist $^{C S}$ animals

158 Overall, it was difficult to visualize vessels at the sinus confluence or along the SSS in Twist $1^{C S}$ mice

159 using chromogenic staining and a stereomicroscope. We therefore sought to develop alternative,

160 high-resolution methods for imaging meningeal lymphatic networks (Fig. 2D, E, Fig. S3). To image

161 dorsal networks along the TVS and SSS, we adapted a previous method that involved removing the

162 dorsal half of the skull, above the temporal bone, with the meninges still attached (Louveau et al.,

163 2018b). The skullcaps were post-fixed and decalcified to remove auto fluorescence, and the tissue

164 was stained using free-floating immunohistochemistry. Incisions were made along the corners of the

165 frontal and occipital bones and the tissue was flat mounted onto slides. This technique provides

166 superior resolution compared with chromogenic immunohistochemistry (Fig. 2D) and allows the

167 option for 3D reconstructions.

168 We used this high-resolution technique to determine if mLV development was affected in juvenile

169 animals at postnatal (P) day 16 when mLVs are actively growing and remodeling along the TVS. The

170 TVS was visualized using alpha-smooth muscle actin (aSMA) to mark the thin layer of smooth

171 muscle that envelopes the venous endothelium, and mLVs were stained with Lyve-1. In controls,

172 meningeal lymphatic networks exhibited numerous sprouts along the TVS (Fig. 3A). By contrast,

173 networks in Twist $1^{C S}$ animals mostly consisted of long, unbranched vessels that were devoid of

174 sprouts, and coverage along the TVS was reduced (Fig. 3B, n=3). In severely affected adult animals,

175 the lymphatic networks were markedly hypoplastic along the TVS and SSS and largely missing

176 around portions proximal to the confluence. aSMA staining revealed that hypoplastic tissue

177 surrounding the confluence and underlying regions of bone loss contained myofibroblasts, suggesting

178 it was fibrotic. Tangled bundles of blood vessels were commonly observed and Lyve-1 positive 
macrophages were abundant. These macrophages normally reside in the leptomeninges (Mundt et al.,

180 2019; Van Hove et al., 2019), and their presence suggests arachnoid tissue remained attached to the

181 skull and thin layer of hypoplastic dura in more severely affected Twist $1^{C S}$ animals. Venous smooth

182 muscle coverage appeared patchy in severely affected animals compared to controls, and aSMA-

183 positive cells were sometimes present in tissue surrounding the proximal portions of the TVS

184 adjacent to the SgS and PSS, suggesting they failed to migrate (Figs. 3C, D). Interestingly, at the

185 sinus confluence, hyperplastic lymphatic vessels were sometimes observed (Fig. 3D). Overall, these

186 results indicate that the growth and sprouting of $\mathrm{mLVs}$ is affected in Twist $1^{C S}$ animals, accounting for

187 loss of meningeal lymphatic networks in adult animals.

188 Basal meningeal lymphatic networks are variably affected in Twist $^{C S}$ animals

189 Although dorsal skull flat mounts provide excellent resolution of vessels along the SSS and TVS,

190 basal networks present along the SgS and PSS were often damaged during the procedure. We

191 therefore analyzed basal meningeal lymphatic networks using a variation of the aforementioned

192 technique (Fig. S3). The skull was harvested and bisected along the dorsal midline, treated with the

193 same post-fixation and decalcification conditions, and excess muscle, fat, hair and connective tissue

194 were removed. The dural tentorium was cut off, the optic nerves were removed from their sheathes,

195 and incisions were made on the corners of the rounded skull to permit flat mounting of the softened

196 tissue onto a slide. The temporal bone was carefully removed in order to not disrupt basal lymphatic

197 networks that reside along the SgS and PSS, which are located along the dorsal and lateral margins of

198 this bone, respectively (Fig. S3). In some animals, it was necessary to thin the skull by peeling off the

199 outermost layer of the calvarial bone.

200 These "basal preparations" fully preserve mLV networks along the SgS and PSS, and also allow

201 imaging of mLVs along arteries near the skull base. Moreover, the vessels along the TVS are also

202 preserved, but care must be taken to preserve networks at the lateral edges of the sinus confluence.

203 Overall, both techniques provide superior resolution to dural flat mounting. They are not subject to

204 tears and creases that can occur from handling the thin dural tissue that has been scraped from the

205 skull, and perfectly preserve the 3D architecture of the lymphatic networks. For these analyses, we

206 crossed Twist $1^{C S}$ animals with a Prox ${ }^{\text {tdTomato }}$ BAC-transgenic reporter line that provides bright red

207 fluorescence in mLVs (Fig. 4A)(Hong et al., 2016). 
In mildly affected animals, growth along the TVS was more comparable to control littermates as branched networks were observed at "hotspot" regions; however, vessel growth was still absent in areas where the TVS was missing (Fig. 4B, B'). Interestingly, basal networks along the PSS were

211 exuberant in these mildly affected animals, containing long vessels with numerous branches (Fig. 4B

$212 \mathrm{n}=2$ ). In moderately affected animals, hypoplastic networks with rudimentary "hotspots" and less

213 branching were present along the intact TVS, but were largely absent and/or atrophic on the side

214 where the TVS was missing (Fig. 4C, C', n=3). Basal lymphatic networks along the PSS were less

215 affected than dorsal networks. Long vessels were observed but branching and complexity was

216 reduced compared to more mildly affected animals and controls. In severely affected animals $(n=2)$,

217 mLV growth and complexity along the TVS was significantly affected, and few if any sprouts and

218 branches were observed. mLVs were also present along ectopic veins that branched from the

219 remaining segments of the TVS (Fig. 4D). Basal networks along the PSS were variably affected.

220 Similar to mild/moderately affected animals, long unbranched vessels were sometimes observed, and

221 disorganized hyperplastic networks could accompany these on the contralateral side (Fig. 4D). In

222 general, animals that were missing dorsal networks had exuberant basal networks, suggesting they

223 were compensating for vessel loss along the TVS. The growth of mLVs along the sigmoid sinus was

224 also more robust in severely affected Twist $1^{C S}$ animals versus controls (Fig. 4D). Finally, vessels

225 along the PPA and MMA were mildly affected; coverage along distal, dorsal portions of the MMA

226 towards to skull apex appeared reduced in regions where dura was hypoplastic (Fig. S4).

\section{Lymphatic drainage to the deep cervical lymph nodes is reduced in TwistI $^{\text {CS }}$ animals}

228 Meningeal lymphatics drain macromolecules and waste from CSF, and facilitate the trafficking of 229 immune cells to the dcLNs (Louveau et al., 2018a). Although the main collection point of meningeal 230 lymphatic drainage is the dcLNs, these vessels also drain to the superficial cervical lymph nodes

231 (scLNs), albeit to a lesser extent (Aspelund et al., 2015). To determine if drainage to the dcLNs was 232 affected, we infused a $45 \mathrm{kDa}$ Ovalbumin-647 tracer into the CSF by accessing the subarachnoid 233 space through the cisterna magna. We selected mild/moderately affected animals in which the

234 meninges and subarachnoid space were preserved. The amount of tracer in the dCLNs was

235 significantly reduced in Twist $1^{C S}$ animals (Fig. 4E), whereas the amount of tracer in the scLNs was 236 comparable (data not shown). In addition, the dcLNs showed unilateral hypoplasia in some animals, 237 suggesting lymphatic drainage was ipsilateral or passaged through alternative routes. These results 
suggest that both the growth and functions of meningeal lymphatic networks are compromised in craniosynostosis.

240 Our results show that changes to the local meningeal environment are sufficient to disrupt the growth

241 and remodeling of mLVs in craniosynostosis. The model we have chosen for our current analysis is

242 overall more affected than what is typically observed in humans, and shows similarities with

243 Sweeney-Cox syndrome, a severe craniofacial disorder caused by dominant-negative mutations in

244 TWIST1 (Kim et al., 2017; Takenouchi et al., 2018). However, homozygous Twist ${ }^{F L X / F L X}$ :Sm22a-

245 Cre animals model venous malformations that are absent in heterozygous Twist ${ }^{\text {FLX/WT }}$ :Sm22a-Cre

246 animals, the latter of which more closely mimic skull phenotypes found in humans (Tischfield et al.,

247 2017). Furthermore, more mildly affected animals with bilateral coronal suture fusion approximate

248 the human condition and Twist $1^{F L X / W T}:$ Sm22a-Cre heterozygous animals. Thus, the lymphatic

249 phenotypes we describe here are likely to be present in humans to varying degrees, especially those

250 who have more severe forms of craniosynostosis with loss or hypoplasia of the dural venous sinuses.

251 Work is underway to investigate meningeal lymphatic phenotypes in heterozygous

252 Twist $1^{F L X / W T}$ :Sm22a-Cre animals, and other forms of craniosynostosis caused by activating gene

253 mutations in FGFR2 (Johnson and Wilkie, 2011).

254 The growth of mLVs is dependent upon Vegf-c, which is expressed by venous and arterial smooth 255 muscle in the meninges (Antila et al., 2017). In agreement, mLVs were rarely observed in regions where the TVS was missing in affected animals, whereas vessel growth was observed along ectopic veins that developed in the absence of proper venous sinuses. This suggests smooth muscle derived growth factor signaling from these vessels was sufficient to induce lymphatic growth. Smooth muscle coverage appeared normal on the middle meningeal arteries (which are preserved in these animals), and we did not observe overt changes to meningeal lymphatics that grew alongside these vessels, with the exception of the more dorsal portions of the MMA where dura was hypoplastic. Thus, some of the observed changes to lymphatic networks may be attributed to venous malformations and attenuated Vegf-c signaling. In addition, hypoplastic dura and loss of the extracellular matrix is likely to affect the development of lymphatic networks. Notably, the development of dermal lymphatic networks in mid-gestation embryos relies on extracellular activation of integrin- $\beta 1$, which can bind and activate Vegfr3 independent from Vegf-c (Planas-Paz et al., 2012). Also, the migratory abilities of dermal lymphatics in mid-gestation embryos are influenced by mechanical forces regulated by tissue stiffness (Frye et al., 2018), which may be altered in more severely affected animals with 
hypoplastic, fibrotic dura. Thus, mLV phenotypes in Twist $1^{F L X / F L X}: S m 22 a-C r e$ animals may manifest from combinatorial changes to the surrounding environment that impinge upon Vegfr3 activation, and the growth and sprouting of lymphatic networks. Future studies will examine changes to these processes in our models.

273 In even mildly/moderately affected Twist $1^{F L X / F L X}:$ Sm $22 a-C r e$ animals, lymphatic drainage to the dcLNs was significantly diminished. This can be expected to affect immune cell trafficking as well as CNS waste clearance, especially because ablating mLVs affects the functions of the brain's perivascular waste clearance system (i.e. the glymphatic system) (Da Mesquita et al., 2018; Louveau et al., 2017). Impaired CNS waste clearance is associated with the accumulation of amyloid-beta plaques and cognitive impairment (Iliff et al., 2012; Da Mesquita et al., 2018), whereas altered meningeal immune cell trafficking can also affect brain function and behavior in addition to neuroinflammatory processes (Louveau et al., 2018a; Alves de Lima et al., 2020). In aging animals, meningeal lymphatic networks also naturally deteriorate; dorsal vessels regress whereas basal vessels become hyperplastic (Ahn et al., 2019). Interestingly, hyperplastic basal networks were seen in a subset of animals in the present study. Notably, it is unknown if craniosynostosis may be associated with a higher prevalence of Alzheimer's disease or other forms of neurodegeneration that lead to cognitive decline and dementia. Likewise, to our knowledge, changes to neuroinflammatory processes have not been reported or studied in craniosynostosis. Given that craniosynostosis is associated with venous hypertension and impaired CSF drainage, our new findings imply that many individuals with craniosynostosis, especially syndromic forms, may unknowingly be at heightened risk for cognitive decline due to cerebrovascular malformations, impaired waste clearance, and/or changes to neuroinflammatory processes resulting from altered immune surveillance.

291 In the present study, we have developed new methods to facilitate the characterization of meningeal

292 lymphangiogenesis. Although dural scrapings provide a fast and cost-effective method for visualizing mLVs, this method may not be suitable for all models, especially those possessing meningeal malformations. Our innovative skull flat mounting technique is a superior alternative, as it perfectly preserves meningeal lymphatic networks and provides high resolution imaging in their native environment. Moreover, it does not suffer from imaging artifacts that arise from rips, creases, and folding which may occur from scraping dura from the skull and/or flat mounting the tissue onto 
best techniques for studying mLVs may depend upon the specific animal model(s) and the types of questions that need to be addressed.

301 Figure 1: Twist ${ }^{C S}$ animals have craniosynostosis and hypoplastic meninges. (A) Sm22a-Cre is active in sutural mesenchyme, as detected by the presence of red fluorescent protein (Rosa26:Ai14 $\left.{ }^{\text {tdTomato }}\right)$. (B) Representative skull phenotype from a moderately affected Twist $1^{C S}$ animal. Arrowheads denote fusion of the coronal and lambdoid sutures. (C and D) Coronal section through the heads of an e16.5 control (C) and severely affected Twist $1^{C S}$ embryo (D). Fibronectin (red) marks the meningeal extracellular matrix, which is markedly hypoplastic in severely affected embryos. (E and F) Coronal sections through the head of an e16.5 control (E) and a mildly affected Twist $1^{C S}$ embryo (F) stained with hematoxylin and eosin. The meninges are preserved but hypoplastic periosteal dura and condensed osteogenic mesenchyme are observed at the dorsal midline and not well separated from the overlying dermis (dm, arrowheads). Dashed lines denote SSS

311 hypoplasia. (G-I) e16.5 coronal sections adjacent to the dorsal midline from a control (top row) and

312 mildly affected Twist $1^{C S}$ embryo (bottom row). (G) The dura extracellular matrix is hypoplastic

313 according to fibronectin staining (arrowheads). Connexin-43 (H) and e-cadherin (I) staining show the

314 arachnoid membrane is intact. Bone (b) development, marked by n- and e-cadherin, is delayed and/or

315 absent. $\mathrm{SS}=$ sagittal suture, $\mathrm{CS}=$ coronal suture, $\mathrm{LS}=$ lambdoid suture, $\mathrm{d}=\mathrm{dura}$, br=brain. Scale bars

316 (A) $2.5 \mathrm{~mm}$, (C) $1 \mathrm{~mm}$, (E) $40 \mu \mathrm{m}$.

317 Figure 2: Dorsal meningeal lymphatic networks are affected in wwist $^{C S}$ animals. Dorsal 318 craniotomies from a P60 control (A) and moderately affected Twist ${ }^{C S}$ animal (B). mLVs are 319 depicted by chromogenic Lyve-1 staining (brown). Branched networks are present at the sinus 320 confluence in controls (A', arrowheads), and are missing in Twist $1^{C S}$ animals (B', arrowheads).

321 Vessels along the TVS are hypoplastic in Twist $1^{C S}$ and do not extend to the sinus confluence

322 (arrowheads, middle panel), and vessel loss is noted along the SSS (arrowheads, bottom panel). (C)

323 Dural scraping from a control animal expressing Sm22a-Cre and the Rosa26:Ai14 ${ }^{\text {tdTomato }}$ reporter.

324 Sm22a-Cre activity is detected in dura and vascular smooth muscle, but is absent in Lyve-1 positive 325 mLVs (green). (D) Lymphatic networks in ear skin are normal in Twist $1^{C S}$. (E) Traditional method

326 for visualizing mLVs by scraping the dural membrane from the skull. (F) Representative example of 327 the dorsal skull flat mounting method. The Prox $I^{\text {tdTomato }}$ reported allele marks mLVs in red.

328 TVS=transverse sinus, $\mathrm{SSS}=$ =superior sagittal sinus, $\mathrm{SgS}=$ =sigmoid sinus, $\mathrm{PSS}=$ petrosquamosal sinus, 
pg=pineal gland, CS=coronal suture. Scale bars, (C) $500 \mu \mathrm{m}(\mathbf{F}) 1 \mathrm{~mm} . * * p<0.001, * * * p<0.0001$, student's t-test. control (A) and moderately affected Twist ${ }^{C S}$ animal (B). Developing lymphatic networks show numerous sprouts along the TVS in controls, and significantly less in Twist ${ }^{C S}$. Network expansion is reduced according to percent area coverage at P16 ( $n=3$, each side of TVS). Right diagrams illustrate the development of meningeal lymphatic networks along the venous sinuses at P16, and mature networks at P60. (C) Mature lymphatic networks along the TVS in a P90 control and severely affected Twist ${ }^{C S}$ animal (D). mLVs are largely absent in the affected animal, and only a few vessels are present along the initial segments of the TVS (yellow arrowhead). Smooth muscle coverage along the TVS is patchy and $\alpha$-SMA positive cells are seen in adjacent dura (asterisk). Ectopic blood vessels are present and $\alpha$-SMA positive myofibroblasts surround the sinus confluence (SC). Hyperplastic lymphatic vessels are sometimes present at the sinus confluence (sc) in severely affected animals (white arrows) along with Lyve-1 positive macrophages.

344 Figure 4: Basal lymphatic networks are variably affected in Twist $^{C S}$ animals. (A) Basal preparation depicting RFP labeled mLVs in a control Twist $1^{\text {FLX/FLX }}$ :Prox $1^{\text {tdTomato }}$ adult. Boxed regions denote dorsal networks along the TVS and basal networks along the PSS. Left and right panels show mLVs present on each half of the bisected skull. Dorsal vessels along the TVS show extensive branching at "hotspot" regions (A'). Basal networks along the PSS also show increased complexity as shown in right panel (A'). (B-D) mLV networks in mildly affected (B), moderately affected (C), and severely affected (D) Twist1 ${ }^{\text {CS }}$ :Prox ${ }^{\text {tdTomato }}$ animals. In mildly affected animals, networks along the TVS appear normal, but mLVs are missing on the side with segmental loss of the TVS (B'). Basal lymphatic networks show excessively long vessels with numerous branches (B''). Lymphatic networks along the TVS in moderately affected animals are hypoplastic and less complex (compare A' and $\mathbf{C}^{\prime}$ ) with loss of "hotspots". As shown in (C'), basal networks are variably affected but typically less complex with fewer branches, and abnormally long vessels can be present. In severely affected animals, networks along the TVS are extremely hypoplastic and/or missing. Lymphatic vessels are present along ectopic veins (arrowhead in D). Basal networks show variable affection. In this animal, the left side shows long, unbranched vessels, whereas the network on the right is hyperplastic and disorganized. Quantifications for dorsal networks correspond to those vessels 
360 present in the boxed TVS region in panel A, and basal networks correspond to vessels present in 361 boxed PSS regions, including those present along the SgS. (E) The amount of tracer is reduced in

362 Twist ${ }^{C S}$ dcLNs (right) following infusions via the cisterna magna. Scale bar= $1 \mathrm{~mm}(\mathrm{~A})$ and $200 \mathrm{um}$ $363(\mathrm{E})$.

364 Supplemental Figure 1: Skull development in a severely affected Twist $^{C S}$ animal. Loss of 365 mineralized bone is more extensive in severely affected Twist ${ }^{C S}$ animals. In this animal (P60), large 366 gaps of non-ossified tissue are found in regions where the coronal (cs), frontal (fs), and sagittal 367 sutures (ss) normally develop. Meningeal hypoplasia is also more severe in these animals, as seen in

368 figure $1 \mathrm{~d}$. The length of the skull, as measured from the frontal bone to the occipital bone (dashed 369 line), is reduced by approximately $25 \%$ in Twist $1^{C S}$ animals. *** $p<0.0001$, students t-test.

Supplemental Figure 2: Twist ${ }^{C S}$ animals have hypoplastic dural venous sinuses with segmental loss of the TVS: Dorsal skull flat mounts from a P16 control (left) and Twist $1^{C S}$ animal (right). The dural venous sinuses and mLVs are visualized according to alpha-smooth muscle actin (red) and Lyve-1 (green) staining, respectively. All Twist ${ }^{C S}$ animals have unilateral or segmental loss of the TVS (arrowheads). The intact TVS is typically hypoplastic and tortuous and the SSS is hypoplastic in all animals. Lyve-1 macrophages surround the sinus confluence in moderate/severely affected animals. Note the absence of lymphatic vessel sprouting along the intact TVS and contralateral remaining segment in the Twist $1^{C S}$ animal compared to the control. (1) Following immunohistochemistry, carefully gross the basal sample by cutting off the nasal mucosa, maxilla, and mandible. Next, remove the cartilage, muscle, fat, and excess connective tissue on the outer surface of the skull. If done well, you should receive results similar to (2). Make sure to clean the outer cranial surface with care so to not tear or puncture decalcified skull bone, as this will damage the meninges inside of the skull. (2) Use micro-scissors to cut off the tentorium and remove the optic nerve sheath inside the cranium. Perform this procedure under a fluorescent microscope. Switch between bright field and epifluorescence to plan your cuts and prevent accidental damage to the mLVs. On the outside of the cranium, cut or peel off the zygomatic arch. Results should approximate (3). (3) Inspect work and adjust cuts/cleaning as necessary. (4) While switching between fluorescence and bright-field, cut out areas of the occipital (ob), parietal (pb), and frontal bones (fb) without disturbing the mLVs, as indicted by the red dashed lines. (5) Use micro-scissors and extreme care to remove the inner ear (ie) bone. Switching between fluorescence and bright-field will help 
prevent damage to mLVs. (6) Inspect work and adjust cuts/cleaning as necessary. (7) Place tissue on a slide with the meningeal side (inner cranium) facing up, and create a large covering of mounting media around the sample. This can be done by withdrawing $600 \mu 1$ of mounting media. Pipet 200$300 \mu 1$ on the slide and overlay the sample. Then place $300-400 \mu 1$ of mounting media on top of the sample. (8) Apply coverslip, allow to dry, and image. Samples can be refrigerated and stored long term.

Supplemental Figure 4: $\mathrm{mLV}$ coverage along the middle meningeal artery is mildly reduced in

Twist ${ }^{C S}$ : Prox $I^{\text {tdTomato }}$ labeled mLVs along the pterygopalatine (PPA) and middle meningeal arteries MMA towards the skull apex is patchy and reduced in Twist $1^{C S}$ animals (arrowheads). Otherwise, arterial $\mathrm{mLV}$ coverage is relatively normal in Twist $^{C S}$ animals.

\section{Materials and Methods:}

Animals: The following transgenic mice were used: Twist $1^{F L X}$ (RRID:MMRRC_016842-UNC), Prox $1^{\text {tdTomato }}$ (RRID:MMRRC_036531-UCD), Rosa26:Ai14 ${ }^{\text {tdTomato }}$ (RRID:IMSR_JAX:007914). For

all experiments, male and female mice were included. Animals were maintained on a mixed genetic background (C57B1/6;FVB;CD1). Embryos obtained from timed matings were considered 0.5 days old upon observance of a plug. Experiments were approved and carried out under IACUC protocol

Antibodies: The following antibodies were used: Lyve-1 (1:300, Abcam, ab14917, RRID:AB_301509), RFP (1:1500, Rockland, 600-401-379, RRID:AB_2209751), Fibronectin (1:250, Abcam, ab2413, RRID:AB_2262874), aSMA (1:300, Sigma, C6198, RRID:AB_476856). Nuclei were visualized using Hoechst staining (1:2000, Thermofisher H3570).

413 Immunohistochemistry: Heads from E16.5 embryos were decapitated and fixed overnight in 4\%

414 PFA at $4^{\circ} \mathrm{C}$. The heads were then washed and dehydrated in steps of $50 \%$ and $80 \%$ ethanol prior to 415 paraffin embedding. $10 \mu \mathrm{m}$ paraffin sections were collected for staining and images were obtained 416 using a LSM700 confocal microscope with a 20x 0.8 NA objective. For chromogenic 3,3'417 diaminobenzidine (DAB) staining, the SignalStain DAB substrate kit was used (Cell Signaling Cat\# 418 8059) according to manufacturer's instructions. Briefly, following application of the primary 419 antibody (Lyve-1), 800 $\mu 1$ of ant-rabbit horseradish peroxidase was added to the excised dorsal skull 420 tissue for 30 minutes in a 24 well plate at room temperature. $30 \mu 1$ of DAB was added to $1 \mathrm{ml}$ of the 
421 DAB substrate solution. The solution was added to the tissue and allowed to incubate for 2-4 minutes

422 until the signal was detectable but not saturated. The solution was then discarded and the tissue was

423 washed in distilled water.

424 Dorsal and basal skull flat mount preparations: The animals were perfused with $4 \%$

425 paraformaldehyde, heads decapitated, and all skin and musculature were removed from the dorsal

426 half of the skull. The lower jaw was removed by inserting scissors into the oral cavity to cut the

427 mandible and attached muscles. Angled micro scissors were used to cut laterally from the cisterna

428 magna along the temporal bone to each orbit, and the nasal bones were severed to isolate the

429 calvarium. The tissue was post-fixed in $2 \%$ PFA overnight at $4^{\circ}$ Celsius, washed in PBS, and then

430 treated with Dent's Fix (80\% methanol, 20\% DMSO) overnight at $4^{\circ}$ Celsius. Skulls were next

431 decalcified in 14\% EDTA for seven days at $4^{\circ}$ Celsius and then cleaned of fat, muscle, and

432 connective tissue. Afterwards, the skulls were incubated with primary antibodies for four days,

433 followed by secondary antibodies for two days at $4^{\circ}$ Celsius in $0.5 \%$ triton with $10 \%$ normal goat

434 serum. Incisions were made at the corners of the frontal and occipital bones, and the tissue was flat

435 mounted onto slides in mounting medium (SouthernBiotech Fluoromount-G) and cover slipped.

436 Basal flat mounts, which are described in detail in supplementary figure 3, were otherwise similar to

437 the dorsal flat mount preparation. The skull was bisected along the dorsal midline into halves by

438 making an incision along the occipital bone midline, inserting the tips of scissors into the nasal

439 bones, and opening the scissors to split the skull.

440 Skull 3D X-ray microscopy (computed tomography): Animals were sacrificed via transcardial

441 perfusion with $4 \%$ paraformaldehyde (PFA). The heads were then decapitated and post-fixed

442 overnight in 4\% PFA. Hair and skin were removed prior to imaging. Images were obtained using a

443 Bruker Skyscan 1272 X-ray microscope. The following scan conditions were used: Image pixel

444 size=13.5um, camera=1632 columns $x 1092$ rows, rotation step=0.4 degrees, Frame averaging=3,

445 Filter=1mm Al. The resulting images were reconstructed and converted to dicom format with

446 Skyscan Ctan software. Dicom files were opened in Vivoquant for segmentation of teeth and bone

447 from less dense soft tissues.

448 Microscopy and lymphatic vessel quantifications: Images were acquired on a LSM700 confocal

449 microscope using a 10x 0.3 NA objective. For dorsal and basal lymphatic preparations in figures 3

450 and 4, maximum intensity projections (MIPs) were obtained using $7 \mu \mathrm{m} \mathrm{z}$-stacks. For the

451 quantification of sprouts along the TVS, these were manually counted from three control and 
experimental animals. Values were obtained from vessels present along the left and right transverse sinuses. Each value was imputed separately for statistical analysis ( $\mathrm{n}=3$ animals, six data points). The total vessel length and number of junctions for both the dorsal and basal networks in figure 4 were analyzed from MIPs using the AngioTool plug-in for ImageJ. For dorsal networks, a region of interest was centered over the transverse sinus, extending to the sinus confluence. For basal networks, a region of interest was centered over vessels along both the sigmoid and petrosquamosal sinuses (refer to figure 4a). The following settings were used in Angiotool: Dorsal networks, value diameter and intensity=12 and for basal networks, value diameter and intensity=9, fill small holes $=80$. Values were obtained from at least one half of the skull for vessels comprising dorsal and basal networks from six control and seven experimental animals.

Infusion of molecular tracers into cerebrospinal fluid: Animals were anesthetized using

463 ketamine/xylazine $(100 \mathrm{mg} / \mathrm{kg})$. An incision was made on the midline of the head at the occipital 464 crest. Once the skin was excised, curved forceps were used to break through the superficial connective tissue to reveal the underlying muscle. The muscle was carefully separated along the midline to expose the opening to the cisterna magna, and care was taken to not induce tears or bleeding. A 45kDa ovalbumin-647 tracer (Molecular Probes, Invitrogen) was mixed in artificial cerebrospinal fluid $(20 \mu \mathrm{g} / \mathrm{ml}$ solution), and $5 \mu \mathrm{l}$ of solution was loaded into a $10 \mu \mathrm{l}$ Hamilton syringe attached to polyethylene tubing and injected into the cerebrospinal fluid via the cisterna magna. The solution was injected using a 28-gauge needle and a Nanomite infusion system (Harvard Apparatus) with an injection rate of $2.5 \mu \mathrm{l} / \mathrm{min}$. Animals were kept on supplemental oxygen throughout the duration of the experiment to stabilize breathing and minimize hypercapnia, and the needle was glued in place to prevent depressurization. After 30 minutes had passed, the needle was removed and the animals were immediately euthanized by transcardial perfusion.

475 Cervical lymph node imaging and quantifications: Animals were perfused with 4\% PFA and the superficial and deep cervical lymph nodes were dissected under fluorescence. The tissue was allowed to post-fix for 12 hours in $2 \%$ PFA, prior to sinking in $30 \%$ sucrose and embedding in Neg-50 medium. $20 \mu \mathrm{m}$ sections were cut from three control and three experimental animals. The sections were imaged using a Leica M165FC stereomicroscope equipped with a 1x objective and DFC7000T camera. Ten $20 \mu \mathrm{m}$ sections per animal from the deep cervical lymph nodes were thresholded and analyzed by tracing out the tissue sections and calculating the percent area coverage. The values were 

confocal microscope with a 20x 0.80 NA objective.

Statistics: Statistics were performed using GraphPad Prism 9.0. For all analyses, unpaired student t tests with Welch's correction were performed.

\section{Conflict of Interest}

The authors declare that the research was conducted in the absence of any commercial or financial relationships that could be construed as a potential conflict of interest.

\section{Author Contributions}

491 Conceived and designed project (PA and MAT). Performed experiments and analysed data (PA,

492 MM, MAT). Wrote and edited manuscript (PA, MM, MAT).

\section{$493 \quad$ Funding}

494 Funding was provided by a Busch Biomedical Research Grant (MAT) and the Robert Wood Johnson

495 Foundation (\#74260).

\section{Acknowledgments}

497 The authors would like to thank Young Kwon-Hong (University of Southern California) for 498 providing Prox $I^{\text {tdTomato }}$ mice and the Rutgers Molecular Imaging Center (D. Adler and P.

499 Buckendahl) for assistance with skull 3D x-ray microscopy. Michael Falen and Kush Desai for 500 assistance with mouse dissections and immunohistochemistry. VE Abraira for providing images from 501 BioRender used in figure 3. Marianne Polunas and the Rutgers Research Pathology Services Core for 502 assistance with embryo embedding and hematoxylin/eosin slide preparation.

\section{References}

507 Ahn, J. H., Cho, H., Kim, J.-H., Kim, S. H., Ham, J.-S., Park, I., et al. (2019). Meningeal lymphatic

508 vessels at the skull base drain cerebrospinal fluid. Nature 572, 62-66.

Alves de Lima, K., Rustenhoven, J., and Kipnis, J. (2020). Meningeal Immunity and Its Function in Maintenance of the Central Nervous System in Health and Disease. Annu. Rev. Immunol. 38, 597511620.

512 Antila, S., Karaman, S., Nurmi, H., Airavaara, M., Voutilainen, M. H., Mathivet, T., et al. (2017).

513 Development and plasticity of meningeal lymphatic vessels. J. Exp. Med. 214, 3645-3667.

514 Aspelund, A., Antila, S., Proulx, S. T., Karlsen, T. V., Karaman, S., Detmar, M., et al. (2015). A 515 dural lymphatic vascular system that drains brain interstitial fluid and macromolecules. J. Exp. Med. 516 212, 991-999. 
518 (2018). Functional aspects of meningeal lymphatics in ageing and Alzheimer's disease. Nature 560,

519 185-191.

520 Deckelbaum, R. A., Holmes, G., Zhao, Z., Tong, C., Basilico, C., and Loomis, C. A. (2012).

521 Regulation of cranial morphogenesis and cell fate at the neural crest-mesoderm boundary by

522 engrailed 1. Development 139, 1346-1358. doi:10.1242/dev.076729.

523 DeSisto, J., O’Rourke, R., Jones, H. E., Pawlikowski, B., Malek, A. D., Bonney, S., et al. (2020).

524 Single-Cell Transcriptomic Analyses of the Developing Meninges Reveal Meningeal Fibroblast

525 Diversity and Function. Dev. Cell 54, 43-59.e4.

526 Ding, X. B., Wang, X. X., Xia, D. H., Liu, H., Tian, H. Y., Fu, Y., et al. (2021). Impaired meningeal

527 lymphatic drainage in patients with idiopathic Parkinson's disease. Nature Medicine, 27(3), 411-418.

528 El-Bizri, N., Guignabert, C., Wang, L., Cheng, A., Stankunas, K., Chang, C.-P., et al. (2008).

529 SM22 $\alpha$-targeted deletion of bone morphogenetic protein receptor 1A in mice impairs cardiac and

530 vascular development, and influences organogenesis. Development 135, 2981-2991.

531 Frye, M., Taddei, A., Dierkes, C., Martinez-Corral, I., Fielden, M., Ortsäter, H., et al. (2018). Matrix 532 stiffness controls lymphatic vessel formation through regulation of a GATA2-dependent

533 transcriptional program. Nat. Commun. 9, 1511.

534 Hong, M., Jung, E., Yang, S., Jung, W., Seong, Y. J., Park, E., et al. (2016). Efficient Assessment of 535 Developmental, Surgical and Pathological Lymphangiogenesis Using a Lymphatic Reporter Mouse

536 and Its Embryonic Stem Cells. PLoS One 11, e0157126.

537 Iliff, J. J., Wang, M., Liao, Y., Plogg, B. A., Peng, W., Gundersen, G. A., et al. (2012). A

538 paravascular pathway facilitates CSF flow through the brain parenchyma and the clearance of

539 interstitial solutes, including amyloid $\beta$. Sci. Transl. Med. 4, $147 \mathrm{ra1} 11$.

540 Ito, Y., Yeo, J. Y., Chytil, A., Han, J., Bringas, P., Jr, Nakajima, A., et al. (2003). Conditional 541 inactivation of Tgfbr 2 in cranial neural crest causes cleft palate and calvaria defects. Development $542130,5269-5280$.

543 Izen, R. M., Yamazaki, T., Nishinaka-Arai, Y., Hong, Y.-K., and Mukouyama, Y.-S. (2018).

544 Postnatal development of lymphatic vasculature in the brain meninges. Dev. Dyn. 247, 741-753.

545 Johnson, D., and Wilkie, A. O. M. (2011). Craniosynostosis. Eur. J. Hum. Genet. 19, 369-376.

546 Kim, S., Twigg, S. R. F., Scanlon, V. A., Chandra, A., Hansen, T. J., Alsubait, A., et al. (2017).

547 Localized TWIST1 and TWIST2 basic domain substitutions cause four distinct human diseases that 548 can be modeled in Caenorhabditis elegans. Hum. Mol. Genet. 26, 2118-2132.

549 Lee, S.-W., Choi, K.-Y., Cho, J.-Y., Jung, S.-H., Song, K.-B., Park, E.-K., et al. (2006). TGF-beta2

550 stimulates cranial suture closure through activation of the Erk-MAPK pathway. J. Cell. Biochem. 98, $551981-991$. 
552

553

554

555

556

557

558

559

560

561

562

563

564

565

566

567

568

569

570

571

572

573

574

575

576

577

578

579

580

581

582

583

584

585
Louveau, A., Herz, J., Alme, M. N., Salvador, A. F., Dong, M. Q., Viar, K. E., et al. (2018a). CNS lymphatic drainage and neuroinflammation are regulated by meningeal lymphatic vasculature. Nat. Neurosci. 21, 1380-1391.

Louveau, A., Filiano A.J., Kipnis J (2018b). Meningeal whole mount preparation and characterization of neural cells by flow cytometry. Curr Protoc Immunol. 121(1):e50.

Louveau, A., Plog, B. A., Antila, S., Alitalo, K., Nedergaard, M., and Kipnis, J. (2017).

Understanding the functions and relationships of the glymphatic system and meningeal lymphatics. $J$. Clin. Invest. 127, 3210-3219.

Louveau, A., Smirnov, I., Keyes, T. J., Eccles, J. D., Rouhani, S. J., Peske, J. D., et al. (2015).

Structural and functional features of central nervous system lymphatic vessels. Nature 523, 337-341.

Lundgaard, I., Lu, M. L., Yang, E., Peng, W., Mestre, H., Hitomi, E., et al. (2016). Glymphatic clearance controls state-dependent changes in brain lactate concentration. Journal of Cerebral Blood Flow \& Metabolism, 37(6), 2112-2124.

Mundt, S., Mrdjen, D., Utz, S. G., Greter, M., Schreiner, B., and Becher, B. (2019). Conventional DCs sample and present myelin antigens in the healthy CNS and allow parenchymal T cell entry to initiate neuroinflammation. Sci Immunol 4. doi:10.1126/sciimmunol.aau8380.

Ottelander, B. K. D., Den Ottelander, B. K., Van Veelen, M. C., De Goederen, R., Van De Beeten, S. D. C., Dremmen, M. H. G., et al. (2021). Saethre-Chotzen syndrome: long-term outcome of a syndrome-specific management protocol. Developmental Medicine \& Child Neurology 63, 104-110.

Planas-Paz, L., Strilić, B., Goedecke, A., Breier, G., Fässler, R., and Lammert, E. (2012).

Mechanoinduction of lymph vessel expansion. EMBO J. 31, 788-804.

Takenouchi, T., Sakamoto, Y., Sato, H., Suzuki, H., Uehara, T., Ohsone, Y., et al. (2018). Ablepharon and craniosynostosis in a patient with a localized TWIST1 basic domain substitution. Am. J. Med. Genet. A 176, 2777-2780.

Tischfield, M. A., Robson, C. D., Gilette, N. M., Chim, S. M., Sofela, F. A., DeLisle, M. M., et al. (2017). Cerebral Vein Malformations Result from Loss of Twist1 Expression and BMP Signaling from Skull Progenitor Cells and Dura. Dev. Cell 42, 445-461.e5.

Twigg, S. R. F., and Wilkie, A. O. M. (2015). A Genetic-Pathophysiological Framework for Craniosynostosis. The American Journal of Human Genetics 97, 359-377.

Van Hove, H., Martens, L., Scheyltjens, I., De Vlaminck, K., Pombo Antunes, A. R., De Prijck, S., et al. (2019). A single-cell atlas of mouse brain macrophages reveals unique transcriptional identities shaped by ontogeny and tissue environment. Nat. Neurosci. 22, 1021-1035.

Xie, L., Kang, H., Xu, Q., Chen, M. J., Liao, Y., Thiyagarajan, et al. (2013). Sleep Drives Metabolite Clearance from the Adult Brain. Science, 342(6156), 373-377. 


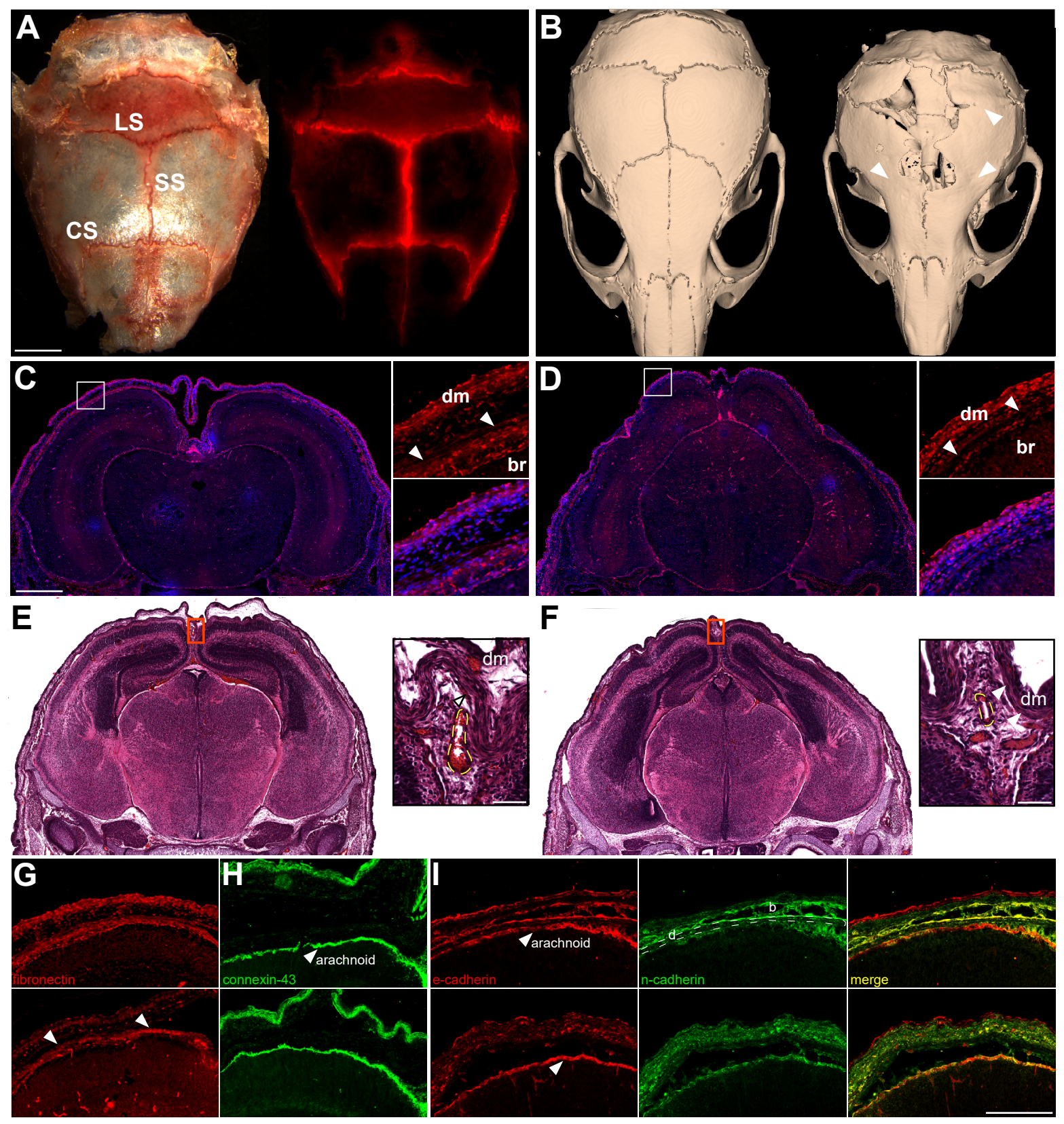



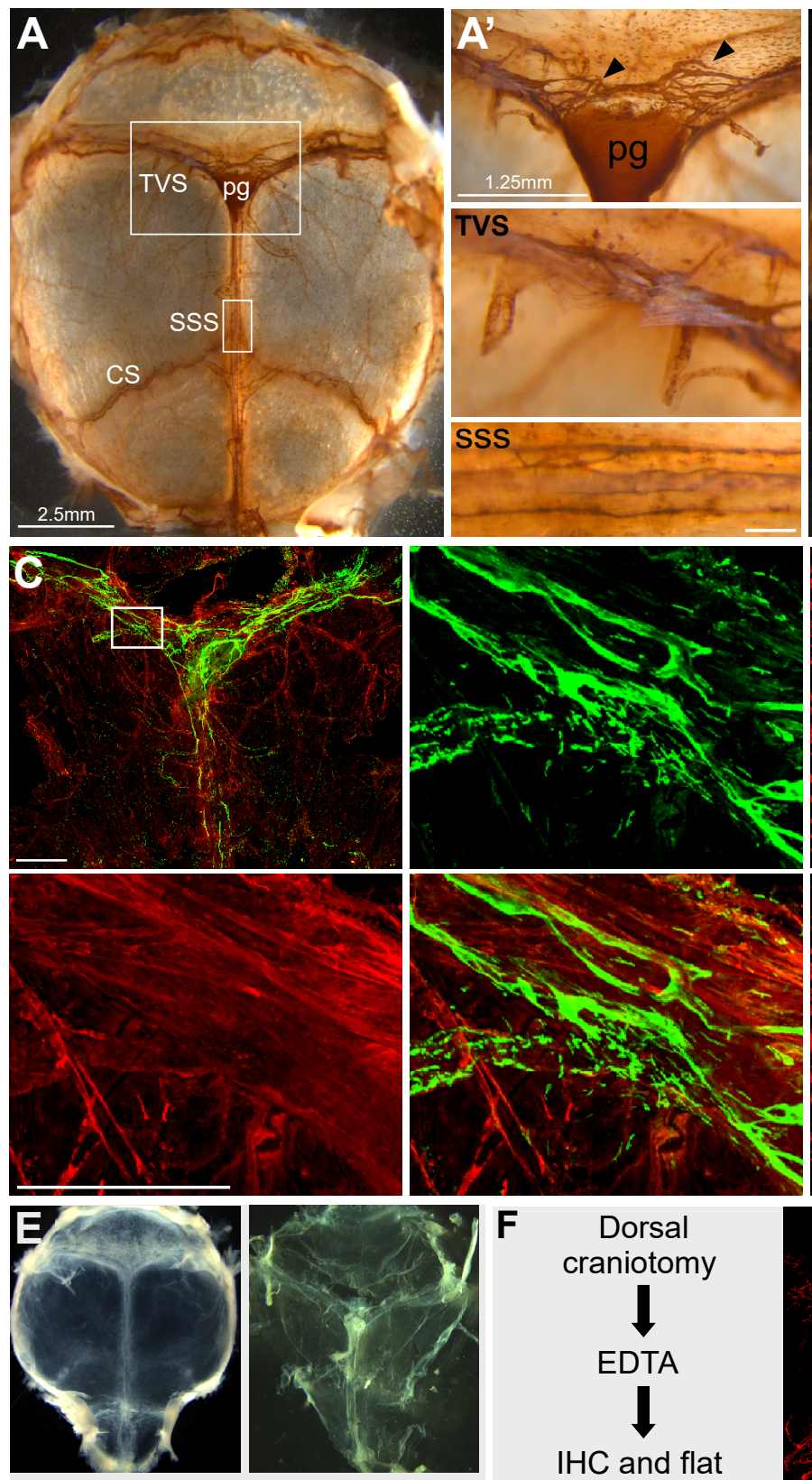

dorsal craniotomy

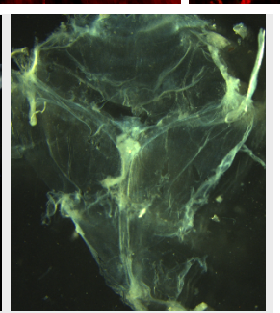

dural scraping
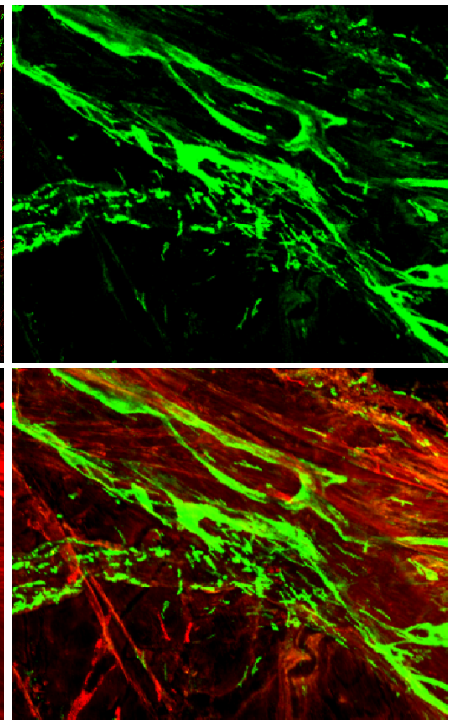

$F$
Dorsal

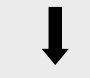

EDTA

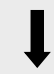

$\mathrm{IHC}$ and flat mounting craniotomy
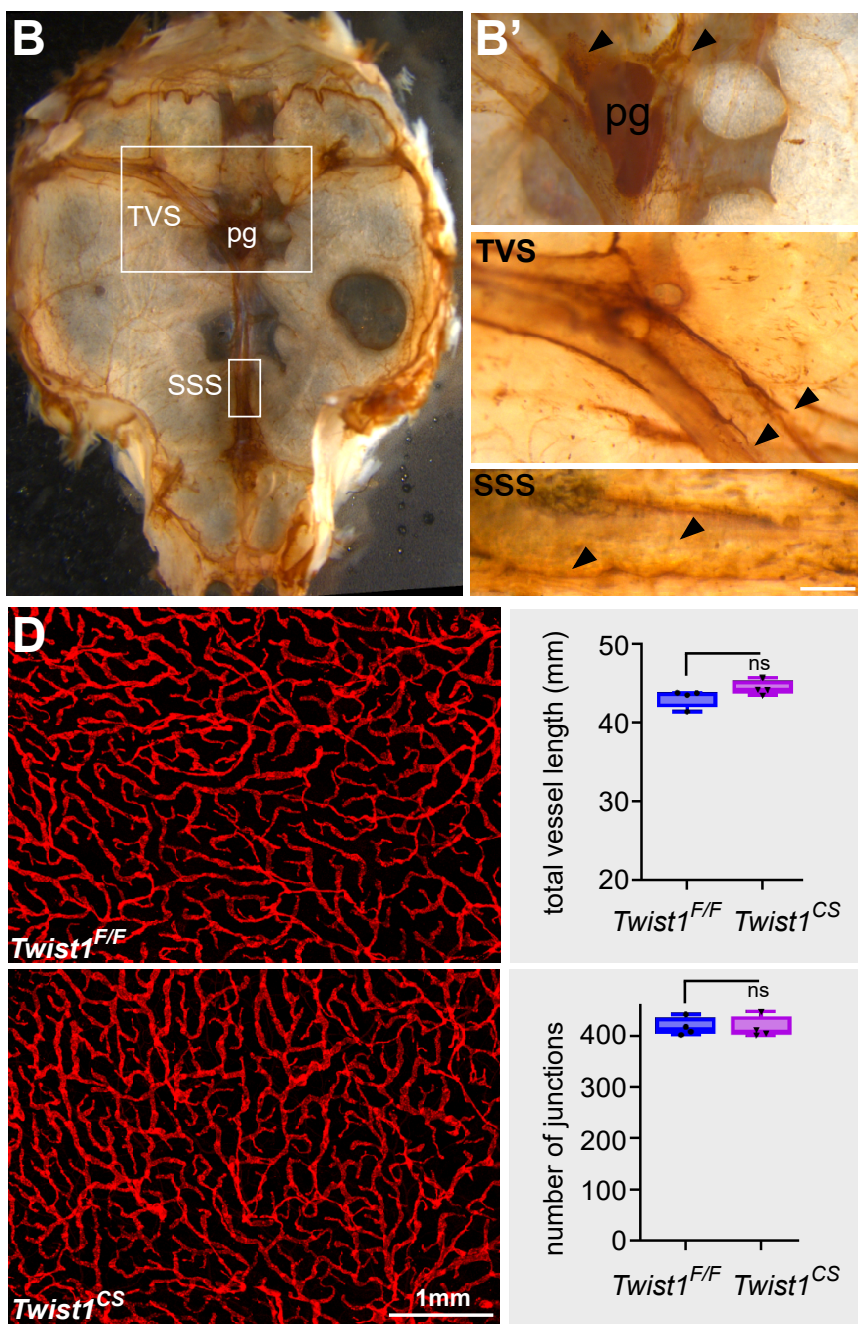

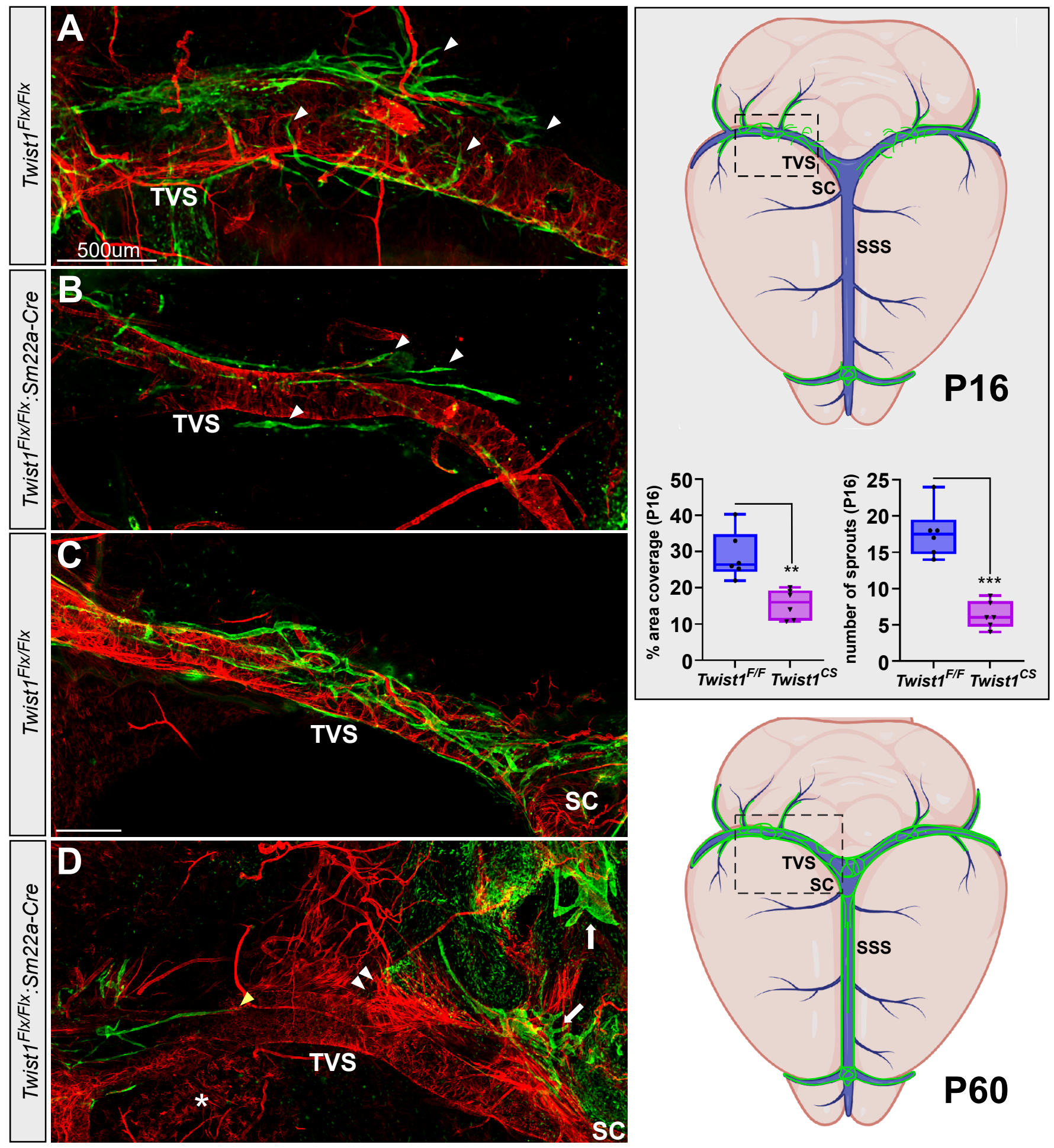

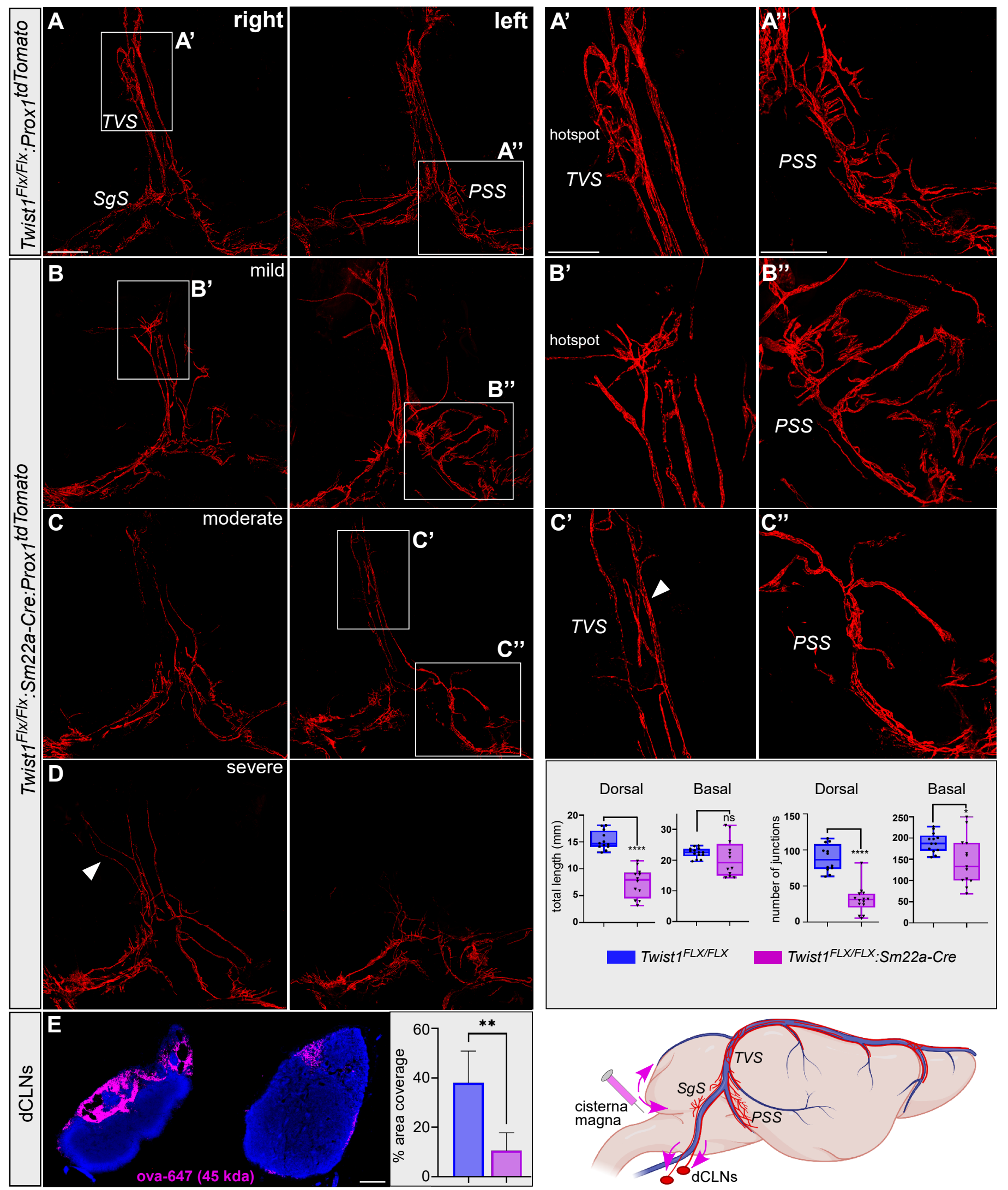